http://dx.doi.org/10.18359/ravi.2670

\title{
La formación investigativa interdisciplinaria de los estudiantes universitarios con el empleo de las tecnologías de la información y la comunicación (TIC) y su dinámica ${ }^{1}$
}

\author{
Mónica Alexandra Pozo Vinueza² Elba María Bodero Poveda ${ }^{3}$ Miguel Alejandro Cruz Pérez ${ }^{4}$ \\ Escuela Superior Politécnica de Chimborazo, Ecuador
}

Recibido, septiembre 16 de 2016

Concepto evaluación, noviembre 15 de 2016

Aceptado, diciembre 27 de 2016

\author{
Referencia: Pozo Vinueza, M. (2017). "La formación \\ investigativa interdisciplinaria de los estudiantes \\ universitarios con el empleo de las tecnologías de la \\ información y la comunicación (TIC) y su dinámica". \\ Revista Academia y Virtualidad, 10, (1), 107-122
}

\section{Resumen}

El presente artículo tiene el propósito de revelar las carencias y limitaciones existentes en el abordaje teórico del vínculo formación investigativa -percibido desde la interdisciplinariedady las tecnologías de la información y la comunicación (TIC), en los procesos formativos universitarios. A partir de los presupuestos teóricos que son objeto de análisis y su reflexión, se aporta una mirada epistemológica donde queda demostrada la existencia de un "vacío teórico" aún no cubierto coherentemente y pendiente por resolver desde la perspectiva de las ciencias pedagógicas, puesto que se trata de que el estudiante universitario, durante el proceso de formación, pueda hacer suyos estos saberes con vistas a la solución de problemas reales de su profesión.

Palabras clave: dinámica, formación investigativa, interdisciplinariedad, proceso de formación.

\footnotetext{
${ }^{1}$ Artículo de revisión.

${ }^{2}$ Especialista en Gerencia de Proyectos; Maestría en Educación a Distancia, E-Learning; PhD en Ciencias Pedagógicas; Docente, Escuela de Ecoturismo, Facultad de Recursos Naturales, ESPOCH, Riobamba, Ecuador. monikiur1010@gmail.com

${ }^{3}$ Profesor Titular de la Carrera de Arquitectura e Ingeniería Civil, Facultad de Ingeniería, Universidad Nacional de Chimborazo, Riobamba, Ecuador. elbaboderopoveda@hotmail.com

${ }^{4}$ Estudiante de Maestría en Pedagogía en la Universidad Nacional de Chimborazo, Riobamba, Ecuador. miguelacp90@gmail.com
} 
The cross-disciplinary research training by college students using the information and communications technologies -ICTs- and its dynamics

\begin{abstract}
This paper aims to show some deficiencies and restrictions in the theoretical basis between research training (from cross-disciplinary perspective) and the information and communications technologies -ICTs-, which affect the training processes at the university. Based on theoretical assumptions, i.e. subject to analysis and reflection from an epistemology point of view, we try to demonstrate a "theoretical emptiness" not covered yet and pending to be solved from the pedagogic sciences perspective in such a way that college student during his/her training process may be able to manage such know-how in order to solve real problems of his/her profession.
\end{abstract}

Keywords: dynamics, research training, cross-disciplinary status, training process.

\author{
A formação pesquisadora interdiscipilinária dos estudantes universitários \\ com o emprego das tecnologias da informação e a comunicação (TIC) e a sua \\ dinâmica
}

\title{
Resumo
}

O presente artigo tem como fim de revelar as carências e limitações existentes na abordagem teórica do vínculo formação pesquisadora - percebido desde a interdisciplinaridade- e as tecnologias da informação e a comunicação (TIC), nos processos formativos universitários. A partir dos pressupostos teóricos que são objeto da análise e sua reflexão, aporta-se um olhar epistemológico onde fica demonstrada a existência de um "vazio teórico" ainda não coberto de jeito coerente e não resolvido desde a perspectiva das ciências pedagógicas, uma vez que trata-se de que o estudante universitário, durante o processo de formação, possa apropriar esses saberes com miras à solução de problemas reais da sua profissão.

Palavras chave: dinâmica, formação pesquisadora, interdisciplinaridade, processo de formação. 


\section{Introducción}

Los problemas fundamentales que enfrenta la humanidad, exigen su estudio como un todo, y la universidad, por tanto, debe cultivar la investigación científica desde una visión interdisciplinaria y generadora de las potencialidades que ofertan las tecnologías de la información y las comunicaciones (TIC). Lo anterior significa que las universidades en los momentos actuales demandan una autotransformación tal que les posibilite dar respuestas a las nuevas estructuras en red y generar bases de aprendizaje de alto valor social agregado en los conocimientos que aprenden sus educandos, desde una óptica investigativa interdisciplinaria y basada en el contexto de su utilización.

Según Schiavo y Ruiz (2012), las TIC facilitan enormemente el trabajo de equipos de investigación que involucren las más diversas disciplinas, aun cuando se encuentran dispersos geográficamente, lo cual hace replantear la investigación interdisciplinaria en la educación superior.

Con base en lo anterior, en esta sociedad de la información y el conocimiento y de desarrollo científico tecnológico, constituye un requerimiento indispensable que la universidad se involucre y establezca un entorno expedito para la formación de una cultura interdisciplinaria (Henriques, 2010). El reto mayor, por supuesto, está en llegar de manera efectiva a los procesos de investigación interdisciplinaria en la educación superior, con un correcto empleo de las TIC por parte de los estudiantes. Al respecto, las universidades deben evolucionar y producir cimientos para una formación investigativa interdisciplinaria sustentada en la utilización de dichas tecnologías.

Por consiguiente, las carreras universitarias deberán propiciar una formación de profesionales capaz de actuar en ambientes intensivos de información, con los conocimientos y las habilidades necesarias para la búsqueda y procesamiento duradero de ésta, mediante un empleo óptimo de las TIC. Esta visión podrá dar respuestas sustanciales a las necesidades de formación de los profesionales aptos para una sociedad inmersa en las TIC y, por tanto, conseguir la excelencia y el desempeño competitivo de éstos.

\section{Fundamentación epistemológica en formación investigativa universitaria mediante las TIC desde un enfoque interdisciplinario y su dinámica}

La universidad como institución social tiene la misión de preservar, desarrollar y difundir la cultura universal, nacional y regional. De esta manera, tiene además una influencia significativa en la sociedad en la medida en que esta última va alcanzando un mayor desarrollo; sin embargo, tal propósito no se logra de manera espontánea (Fuentes, et al. 2011). La formación es un aspecto inherente a la condición humana, categoría que es abordada por Horruitiner (2010), Fuentes, et al. (2011) y Pizzul (2013), entre otros.

Estos autores coinciden cuando definen el término formación como un proceso que se desarrolla con el objetivo de preparar a un individuo en determinada carrera o profesión y que se desenvuelve a través de tres dimensiones: la educativa, la instructiva y la desarrolladora. Consecuentemente, se erigen como ideas rectoras en el proceso de formación, el vínculo del estudio con el trabajo y la unidad entre la instrucción y la educación, aseverado por Horruitiner (2010). Desde esta perspectiva, el proceso de formación debe proporcionar a los sujetos determinados saberes, para la resolución de los problemas de la profesión, a partir de la apropiación y aplicación de diversos contenidos (Coll, Sarabia y Valls, 1992; Mauri, 1993; Díaz y Hernández, 2004), para que desde la conexión de los diferentes elementos del saber, construir conocimientos así como desarrollar habilidades y valores, como elementos esenciales de la inserción social de este proceso, por cuanto, el mismo se sustenta en los pilares fundamentales de la educación (citados por Delors, et al. (1996) y emitidos por la UNESCO, 1995).

De este modo, dichos pilares serán (para cada sujeto y en el transcurso de la vida) los 
fundamentos para su desarrollo: aprender a aprender (saber qué, o contenidos declarativos); aprender a hacer (saber hacer, o contenidos procedimentales); aprender a ser y aprender a vivir juntos (saber ser, o contenidos actitudinales). Por tanto, consiguientemente, la formación es un proceso de desarrollo de los recursos personológicos de los sujetos (Ferry, 1997), sustentada en un proceso de aprendizaje donde los individuos se apropian de los contenidos de la profesión, así como desarrollan y perfeccionan permanentemente determinadas capacidades. Por tanto, el aprendizaje es el mecanismo fundamental de la educación, que posibilita el desarrollo de los sujetos desde un enfoque integrador, como proceso y resultado de todo el sistema de influencias educativas del contexto social, identificado con su cultura y con el necesario nivel de reflexión crítica y conciencia de su accionar permanente.

Se requiere entonces que el individuo, durante el proceso de formación, haga suyos los contenidos profesionales mediante un proceso de aprehensión de la cultura, entendido como el desarrollo del individuo, el cual aprende de la realidad del contexto que lo forma (Pozo, 2016). De este modo, la posibilidad de captar esa realidad le permite su autotransformación $\mathrm{y}$, por ende, la transformación del propio contexto.

Relacionado con lo anterior, la aprehensión de la cultura constituye el hecho de percatarse de algo que está presente, a lo que se le agrega que las ideas científicas constituyen las formas de aprehensión humana de las leyes que rigen el movimiento del mundo objetivo. Por su parte, Fuentes, et al. (2011) aseveran que la apropiación de la cultura es un proceso dinámico en que los sujetos (de manera intencional y sistemática) constituyen su cultura, transformando su entorno y la propia dinámica. Es así que la apropiación de la cultura, como proceso, le posibilita a cada sujeto realizar una profundización del contenido sociocultural, a la vez que se revela contradictoriamente en el propio proceso del desarrollo humano (Pozo, 2016).

De este modo, la profundización del contenido sociocultural constituye un proceso basado en una sistematización de la estructura educativo-pedagógica y en una generalización del contenido cultural en los seres humanos: conocimientos, habilidades, valores, que conllevan a una formación diversa, esencial, flexible y se erige en la generalización esencial y la complejidad de los diversos factores que influyen y condicionan el proceso de la propia formación.

Tal es así que para que ocurra la profundización, la apropiación y la aprehensión, se requiere la sistematización, como una categoría esencial, que de acuerdo con la Pedagogía de la Educación Superior (Fuentes, et al. 2011), es el proceso que le imprime un carácter de continuidad y consecutividad a la formación a niveles superiores, en la construcción científica del contenido sociocultural. La sistematización da respuesta a las interrogantes ¿por qué? y ¿para qué?, en tanto tiene como objetivos no sólo la continuidad, sino también la replicabilidad, entendida como el no dejar perder las experiencias de apropiación analítica, desde la propia vivencia de los participantes.

De igual modo, en la sistematización se reconstruyen una o varias experiencias para explicitar o descubrir la lógica del proceso vivido y los factores que han intervenido. De este modo, se produce un primer nivel de conceptualización desde la práctica concreta que posibilita su comprensión y apunta a trascenderla. A propósito del término sistematización, varios investigadores recrean presupuestos epistemológicos acerca del mismo. Tal es el caso de Lanuez, y Fernández; Jara, Martinic, Barmachea, et al.; Antillón, Azócar, Cadena, y García (referidos por Salas, 2008), todo lo cual permite comprender que la sistematización de la formación representa una articulación entre teoría y práctica, que apunta por una parte a mejorar la propia práctica, donde una se enriquece con la otra en un proceso continuo y gradual.

Con relación a lo anterior, particularmente Sánchez (2009) aborda el proceso de formación para la investigación científica de los universitarios y al respecto refiere distintas 
categorías, tales como la sistematización tecnoinvestigativa, precisando que la misma tiene un carácter dinamizador de dicho proceso, al promover, actualizar y sensibilizar a los estudiantes en cuanto al empleo de los recursos tecnológicos idóneos para el desarrollo de la investigación científica. Esta misma autora refiere el llamado contenido tecnoinvestigativo como aquel que abarca los aspectos tecnológicos y de la investigación científica en lo referente a:

- Desarrollo de las habilidades básicas en el manejo de la información (navegar e interactuar)

- Procesamiento de la documentación digital

- Dominio de un grupo de herramientas generales relativas a la búsqueda, revisión, procesamiento y comunicación de la información digital, de tal manera que lo tecnológico se refiere a las TIC y, por ende, a todas las aportaciones asociadas a estas tecnologías.

Ahora bien, a partir de la irrupción de las TIC en la educación, varios autores han analizado el empleo de las mismas en los procesos formativos, particularmente en los universitarios: Pardo (2004), Izquierdo (2004), Sánchez (2009), Encarnación (2010), Tejada (2010), García (2010), Torres (2014), Silva (2014), Rodríguez (2014), Tapia (2015), por solo citar algunos.

Este mirada epistemológica asume de Pardo (2004) lo referido a la dinámica del proceso de formación en la educación superior con el empleo de las TIC, la cual, a decir de dicha autora, se basa en el intercambio constante y la colaboración vía red que se lleva a cabo entre varios sujetos con intereses comunes que participan en el mismo. Dicha dinámica se desarrolla a través de formas o estructuras espacio-temporales flexibles y diversificadas, en dependencia del uso del espacio y el tiempo. Posee como cualidades: la extensibilidad, la flexibilidad y el cambio de roles, las que tienen como base la interactividad (interacción vía red que se establece en todo proceso formativo con el empleo de las TIC).

En el proceso de formación se fomenta una postura investigativa que le posibilita al estudiante enfrentarse a su praxis en el contexto de aprendizaje, lo que implica "[...] el análisis crítico y reflexivo de sus estrategias investigativas y de las posturas éticas y epistemológicas que guían su quehacer" (Hilarraza; 2012, p. 29), por lo que para lograrlo se requiere propiciar desde el proceso formativo trabajos investigativos, la difusión de resultados, la participación en congresos, entre otros. "Con estas actividades se actualiza y genera el conocimiento, se consolida la investigación en la universidad" (Pérez y Marreno; 2014, p. 67).

En la universidad, la investigación adquiere significación al transformarse en un proceso estratégico para la formación de profesionales; por tanto, los estudiantes deben estar en capacidad de enfrentar los retos de una sociedad cambiante y exigente por el incesante avance tecnológico. La formación investigativa se refiere a aquella formación que fomenta la cultura investigativa y el pensamiento crítico $\mathrm{y}$ autónomo que facilita a los profesores y estudiantes acceder a los nuevos avances de la ciencia y a la capacidad de resolver problemas utilizando el conocimiento para la búsqueda, el análisis y su sistematización. De esta manera, es imprescindible en la misma, la adquisición de métodos, técnicas y reglamentos relacionados con la actividad investigativa (Jiménez, 2006). $\mathrm{La}$ formación investigativa entonces se establece como un parámetro de excelencia en el ambiente de la educación superior y es la base para que profesores y estudiantes la utilicen a fin de desarrollar sus investigaciones (Jiménez, 2006); la misma posee singular significación para la adopción de una postura y una cultura científica entre los mismos (Aldana, 2012).

En la actualidad, la formación investigativa universitaria logra su trascendencia en el ámbito de la educación superior al ser incorporada como eje transversal en los planes de estudio, encaminado a la formación de competencias investigativas, promoviendo un desafío a la universidad como institución impulsora y gestora de las transformaciones del entorno 
social, en el que van a ejercer sus empleos los futuros egresados. De este modo, la formación investigativa se debe reconocer como un proceso en continuo perfeccionamiento, favorecido por ideas y prácticas innovadoras, para lo cual los profesores deben poseer pleno dominio didáctico-pedagógico-profesionalinvestigativo y estar aptos para incorporar los adelantos tecnológicos suscitados por la época moderna, en la que han adquirido un lugar preponderante las TIC.

La formación investigativa es un elemento obligatorio en las carreras, como elemento crucial el hecho que cada una de las disciplinas tiene el compromiso de colaborar en la formación de los profesionales desde sus propias particularidades. Al respecto, en la educación superior se debe estimular en los estudiantes a investigar, cuestionar la teoría y la práctica, construir hipótesis, experimentar y buscar la solución a los problemas (Ruiz, et al. 2011).

La formación investigativa precisa de formas de organización que permitan: vivenciar momentos y roles de la actividad científica, la comunicación interpersonal, el debate científico; vincular la teoría y la práctica, la reflexión y regulación metacognitiva, la socialización de vivencias y experiencias, la criticidad constructiva, la proyección científica de alternativas de solución a los problemas de la práctica (Ruiz, et al., 2011) y considerar que los problemas de investigación sean pertinentes para el entorno actual.

Por su parte, González (2011, p. 77) considera que los planes de estudio deberían propiciar la investigación en el proceso de formación, e implementar una interacción continua en los procedimientos investigativos, generando una cultura investigativa "a través de la síntesis y retroalimentación entre la docencia y la investigación".

La cultura investigativa, según López, et al. (2005, p. 77), “[...] comprende organizaciones, actitudes, valores, objetos, métodos y técnicas relacionadas tanto con la investigación como con la transformación de la investigación o de la misma pedagogía". En las universidades, la cultura investigativa se va conformando a partir de la creación de equipos, grupos, comités, centros de investigación, así como redes investigativas que conforman todo un entramado de relaciones alrededor del proceso de formación investigativa universitaria.

A partir de esta posición se considera que la cultura investigativa que se promueve desde el aula de clase se fortalece y sostiene a través de la promoción de investigadores que cultiven sus líneas de investigación y concentren en lo fundamental estudiantes aventajados, permitiendo que los participantes se ubiquen en un alto nivel de investigación, teniendo como resultado las producciones científicas.

Ramírez (2013, p. 5) define la cultura investigativa en la docencia del pregrado como un sistema complejo, atinente a una cultura organizacional; ésta se desarrolla a través de los procesos de enseñanza y aprendizaje, que convergen en el quehacer investigativo en el que intervienen profesores y estudiantes. En este proceso se comparten normativas, actitudes, valores, motivaciones, intereses, saberes, experiencias, estrategias, técnicas e instrumentos. Es un proceso guiado desde un pensamiento reflexivo en la búsqueda del conocimiento, con propósitos comunes que contribuyen a la mejora de las prácticas pedagógicas, traducido además en aportes y acciones, sistematizadas y socializadas, lo cual posibilita la propuesta y constitución de organizaciones o unidades de investigación que distinguen y caracterizan la comunidad académica como científica y social.

De acuerdo con Orrego (1997), el término cultura investigativa se refiere al conjunto de actitudes, comportamientos, actividades del proceso de enseñanza-aprendizaje encaminado a fundamentar la investigación como elemento esencial en el desarrollo científico del profesional. En ese mismo orden de ideas, Restrepo (2001) define la cultura investigativa como el conjunto de organizaciones, actitudes, valores, objetos, métodos y técnicas, todo en relación con la investigación. Además plantea que la misma es iniciada por los profesores en forma individual, pero poco a poco se integran equipos, grupos, comités y centros de investigación y redes que tejen el sistema 
de investigación en la universidad y que conforman las organizaciones.

Lo anterior permite entender que la cultura investigativa en la docencia de pregrado constituye un sistema complejo, encaminado a preparar estudiantes competentes, es decir, con las capacidades para investigar problemáticas de un mundo cada vez más complejo. Se trata de analizar los problemas y darles solución a los mismos de manera integral y con el empleo de los adelantos tecnológicos, en particular los establecidos por la tecnología digital y las redes informáticas como paradigma de las TIC.

Autores como Ávila (2014) plantean que la dinámica investigativa está dirigida a lograr una interacción entre estudiantes, profesores e investigadores, y la formación investigativa posibilita que los participantes en el proceso promuevan espacios de debate de los diferentes puntos de vista que poseen.

De este modo, la formación investigativa universitaria busca desarrollar en los estudiantes competencias encaminadas a pensar de forma crítica y creativa, abstraerse, analizar, discernir y sintetizar, deliberar sobre un objeto de conocimiento desde las categorías teóricas de las distintas disciplinas, contrastar $\mathrm{y}$ verificar el conocimiento y aplicarlo en la práctica, contextualizar las técnicas de investigación, identificar, plantear y resolver problemas, buscar, procesar y analizar información procedente de fuentes diversas, formular y gestionar proyectos.

A lo anterior se añaden las denominadas competencias tecnoinvestigativas (Sánchez, 2009): navegar e interactuar a través de las redes informáticas para la búsqueda, localización, revisión y procesamiento de la información en formato digital; la elaboración de los marcos teóricos; la solución novedosa a los problemas que investigan así como la corroboración de los resultados con otros especialistas. Dicha autora considera la formación para la investigación científica, sustentada en las TIC, como el proceso de obtención de contenidos científicos, como construcción colaborativa de significados y sentidos entre los sujetos en él implicados, aspectos que se asumen en esta investigación.

Del mismo modo, esta investigadora reinterpreta el enfoque histórico cultural de Vigotsky (1987), al reconocer que los contextos socioculturales son dinamizadores de los procesos de formación investigativa, sustentados en las TIC y caracterizados por la participación de sujetos en diversos contextos, con lo que se produce una influencia mutua entre todos, asumiendo la importancia de la interacción social y de la comunicación entre individuos, como un espacio de crecimiento individual y social de éstos.

Por otro lado, se asume en el proceso de formación investigativa, con el empleo de las TIC, lo referido a la mediación tecnológica (Pardo, 2004), reinterpretada por Tapia (2015, p.54) como

[...] mediación didáctica virtual. Esta es entendida como el conjunto de interacciones que se establecen en entornos mediados por dichas tecnologías (sujeto-sujeto; sujetocontenidos-recursos didáctico-tecnológicos y entre estos últimos), con el fin de facilitar el proceso formativo en condiciones de virtualidad, particularmente en el proceso de formación investigativa, con el empleo de las TIC.

Al igual que las interacciones que se establecen entre los estudiantes y el profesor están dinamizadas por instrumentos de mediación que constituyen los procedimientos que intencionalmente emplea el segundo para hacer aprehensibles los contenidos y las situaciones profesionales con las que interactúan los estudiantes, lo cual está dirigido a favorecer la independencia, la participación y la creatividad de estos últimos.

Con relación a las habilidades científicoinvestigativas, las mismas son entendidas por Chirino, et al. (2005), pues dan la posibilidad de solucionar problemas profesionales desde un desarrollo permanente de los saberes actualizados y su demostración desde la realidad profesional.

Teniendo en cuenta lo anterior, resulta pertinente entender que para desarrollar las competencias investigativas en los estudiantes universitarios se necesita el aporte de cada 
una de las disciplinas de una carrera, con una visión integradora, así como de profesores que estén adecuadamente preparados y sean capaces de aplicar metodologías apropiadas que les permitan orientar a los estudiantes en la actividad investigativa. Al respecto, la orientación es una categoría indispensable de todo proceso formativo, y según Fuentes, et al. (2011), es la acción que realizan los profesores de guiar a los estudiantes en el cumplimiento de determinados objetivos en dicho proceso. En el caso de la formación investigativa estaría encaminada a conducir a los mismos en la actividad de investigación científica.

El proceso de formación para la investigación científica de los estudiantes universitarios, desde los presupuestos teóricos de la teoría holístico configuracional de Fuentes, et al. (2011), la cual se asume como un presupuesto epistemológico importante desde nuestra visión, se debe considerar de manera holística, es decir, como un todo, donde se establece un innegable vínculo con lo académico y lo laboral.

En la actualidad, dada la complejidad de los fenómenos y problemas a los que se han de enfrentar los profesionales, la visión sesgada de conocimientos aislados o fragmentados de una sola disciplina, sin vínculo con otras, atenta contra la objetividad y la visión de conjunto en el enfrentamiento de cualquier problemática o situación que se presente en los distintos contextos.

Respecto a lo anterior, la interdisciplinariedad es un elemento imprescindible en los procesos formativos universitarios (Álvarez, 2004), posición con la que concordamos, ya que los conocimientos fragmentados dificultan que el estudiante se familiarice con razonar y examinar los problemas o situaciones, desde la perspectiva total de las diferentes materias o disciplinas. En la literatura científica se encuentran diferentes definiciones sobre la interdisciplinariedad. Autores como Agazzi (2004) y Álvarez (2002) sostienen que la misma ofrece el camino para superar la fragmentación del saber que la especialización parece hacer inevitable.

Otros autores como Godofredo (2008) la consideran como el encuentro entre distintas disciplinas que posibilitan la integración de saberes científicos, descubriendo interrelaciones en los análisis teóricos, diseños metodológicos y en la interpretación de la información, con vínculos previamente establecidos, los cuales evitan que se desarrollen acciones de forma aislada, dispersa o segmentada. Se trata de un proceso dinámico que favorece el encuentro de soluciones a diferentes dificultades de investigación.

La interdisciplinariedad necesita propósitos y procedimientos compartidos, así como, integrar contenidos de manera activa, colaborativa e investigativa (Pozuelos, et ál. 2012), por lo que se concuerda que esa visión de compartir es beneficiosa en el abordaje de situaciones.

La interdisciplinariedad es fundamentalmente un proceso y una filosofía de trabajo, cuya esencia radica en la construcción o producción de conocimientos, en la cooperación orgánica y la flexibilidad entre los miembros del equipo, la comunicación y la desaparición de barreras (Cabero, 2007), el enriquecimiento mutuo de saberes, la exaltación de valores como la solidaridad, la honestidad, la tenacidad, el respeto y confianza mutuos (Perera, 2000).

Lo anterior permite enfrentar dificultades desde una visión múltiple a los problemas complejos de la realidad (López, et al., 2011), donde se diferencia lo necesario de lo superfluo (Álvarez, 2002), al ofrecer soluciones para ello con una visión integrada del mundo (Ander-Egg, y Follari, 1988). Esto no sería factible hacerlo sin la participación de varias disciplinas (Uribe, 2011 y Hayes, 2004), por lo que se considera necesario plantear actividades conjuntas entre varias disciplinas para que los estudiantes comprendan la importancia del trabajo interdisciplinario en la solución integrada de determinado problema profesional.

La interdisciplinariedad es un proceso que representa el vínculo de todo lo existente, el enlace de los procesos y fenómenos, lo disímil pero único. Desde la filosofía marxista, es la expresión de la unidad material del mundo (Blanco, et al., 2011). Evidencia la adquisición 
de los conocimientos desde lo cognitivo integrador (Cabezas, y Meriño, 2011). La interdisciplinariedad es una práctica que redunda en la producción de nuevos conocimientos, que ni niega las disciplinas ni pretende superarlas, pero que supone varios grados de colaboración y cruce entre ellas para lograr mayor pertinencia y alcance.

Al respecto, la colaboración puede tomar forma de síntesis o integración de perspectivas y supone un esfuerzo adicional al de juntar expertos o personas de diversa formación en torno a un mismo problema. Se le mira como un nuevo procedimiento de perfeccionamiento de los procesos formativos a partir de construir conocimientos integrales y completos y con infinidad de posibilidades. Así observamos que estas aseveraciones tienen como base teórica el reconocimiento de la interdisciplinariedad en el abordaje integral de los problemas. De igual modo, para comprender la magnitud del concepto de interdisciplinariedad se precisa diferenciar teóricamente lo que es la multidisciplinariedad, la interdisciplinariedad y la transdisciplinariedad, tomando en consideración las reflexiones que realiza Bernal (2014), sobre estos tres aspectos. Para dicho autor, la multidisciplinariedad es un diálogo "[...] donde cada disciplina se mantiene dentro de su enfoque, métodos, categoríasy especialidad, sinmás compromiso que el de exponer su punto de vista sobre un tema", mientras la interdisciplinariedad, no se limita solo a exponer un punto de vista, sino que, busca "[...] una cierta razón de unidad, de relaciones, de acciones recíprocas y de interpretaciones entre diversas ramas del conocimiento". Sin embargo, la transdisciplinariedad busca trascender mediante la " $[\ldots]$ integración de saberes que generen un saber superior al existente o una nueva ciencia o disciplina científica" (Bernal, 2014, pp.49-50).

Con respecto a lo anterior, se considera apropiado hacer referencia a la interdisciplinariedad como una forma de entender las relaciones que se establecen entre las diferentes disciplinas, lo que permite dialogar entre ellas desde sus respectivos campos de conocimiento, en tanto, si bien los conceptos de transdisciplinariedad y multidisciplinariedad tienen valor dentro del proceso formativo, desde esta mirada epistemológica que se defiende, el empleo de las TIC como elemento que sustenta la formación científica investigativa tiene un carácter esencialmente interdisciplinario, expresado en la búsqueda de los nexos entre las disciplinas que intervienen en la formación de los profesionales. Al respecto, la interdisciplinariedad trata de buscar los puntos de contacto y la colaboración entre las disciplinas, lo cual obedece a un proceso lógico que se ha considerado en la conformación de las disciplinas de una carrera, que toman parte de la vida para su estudio.

La unidad de las disciplinas está dada por su objeto de estudio: el hombre y su formación. Las disciplinas comparten valores y procedimientos comunes y generales, por lo que es tarea de profesores y directivos investigar los nexos que existen entre disciplinas y asignaturas en términos de objetivos generales, contenidos, métodos, evaluación, bibliografía, entre otros aspectos.

Existen modos de integración que deben constituir los núcleos de los contenidos y ofrecen salida a múltiples disciplinas y a temas transversales. En fin, se trata de integrar el conocimiento dentro de los macrosistemas de conocimientos a que pertenece y recomponer así el todo. La singularidad individual no puede comprenderse al margen de la unidad social y menos aún con conceptos y categorías particulares.

La interdisciplinariedad se expresa como resultado de la influencia de los contenidos de enseñanza en la formación humana y la educación del hombre; requiere la colaboración. Lo importante es explicar y hacer ver cómo existen informaciones, conceptos, metodologías, procedimientos, etc., que tienen utilidad y sentido en más de una disciplina (Torres, 1994). Respecto a la interdisciplinariedad, en esta concepción se asume a Fiallo (2012), al considerarla como un proceso y una filosofía cosmovisiva y de trabajo, como una forma de pensar y de proceder para conocer la 
complejidad de la realidad objetiva y resolver cualquiera de los complejos problemas que ésta plantea. En consecuencia, a tono con dicho autor, la interdisciplinariedad es interpretada como cooperación entre varias disciplinas e interacciones que provocan enriquecimientos mutuos, las que pueden ir desde la simple comunicación de ideas hasta la integración mutua de leyes, teorías, hechos, conceptos, habilidades, hábitos, valores que se han de desarrollar, metodologías, formas de organización de las actividades y de las investigaciones.

El progreso del conocimiento tiene mucho que ver con la capacidad de plantear nuevos interrogantes o reformar viejos problemas desde una nueva visión (Jacob, 2015), con el modo de suministrar soluciones a los mismos a partir de teorías más generales y profundas, así como de técnicas más poderosas y precisas. La universidad no puede aportarle al estudiante todo el conocimiento que éste necesita y que ha acumulado la humanidad, por razones de tiempo y volumen de información. El gran reto consiste en enseñar al estudiante a aprender para que el mismo busque el conocimiento de manera independiente, lo seleccione y lo procese según sus necesidades y posibilidades. Es ahí donde desempeñan un rol decisivo las TIC en la formación investigativa interdisciplinaria, porque dichas tecnologías ofrecen la posibilidad de reducir el tiempo de respuesta y retroalimentación al estudiante durante su proceso formativo, haciendo más eficiente la comunicación (ya sea sincrónica o asincrónica). Por otra parte, las TIC propician de manera rápida y a menor costo, la actualización de materiales de estudio, gracias a la disponibilidad digital que se tiene de los mismos; de igual forma se conciben nuevas estrategias tutoriales para facilitar la investigación de manera colaborativa y cooperativa entre los sujetos que intervienen en el proceso formativo.

Por ello la formación investigativa interdisciplinaria con el empleo de las TIC en las instituciones de educación superior es una condición ineludible de estos tiempos, en correspondencia con la actual era tecnológica por la que transita la humanidad. La formación investigativa interdisciplinaria es el soporte que permite el análisis de los fenómenos y procesos como un todo, donde ningún fenómeno o proceso se superpone a los otros. La misma se manifiesta cuando los sujetos son capaces de enfrentar un problema que se encuentra vinculado con varias disciplinas desde una visión integradora (Wojcipchowski, 2004; Polanco, 2015), para lo cual se requiere un conocimiento amplio e integral, así como habilidades y actitudes para tal empeño.

Consecuentemente, el pensamiento investigativo interdisciplinario promueve, entre otras cuestiones: la habilidad para cruzar las fronteras disciplinarias con el fin de crear conexio $\neg$ nes significativas entre disciplinas; para sintetizar e integrar los conocimientos y los métodos de dis-tintas disciplinas a un problema específico; para pensar y abordar los problemas a través de diferentes disci $\neg$ plinas y desde distintas ópticas; para contextualizar el conocimiento de manera que permita una visión integradora de los elementos que afectan un problema; para resolver problemas y preguntas que no se pueden abordar satisfactoriamente desde una sola disciplina; para trabajar colaborativamente con sujetos de distintas disciplinas, así como, la independencia de pensamiento, el cual no se centra en un solo método ni sigue procedimientos rutinarios, sino que integra las herramientas necesarias para la resolución más comprensiva de un problema; y de la misma manera, para compartir experiencias y conocimientos basados en la reflexión y participar en procesos de construcción y multiplicación de conocimientos con liderazgos compartidos.

Una vez entendida la interdisciplinariedad como la cooperación, la colaboración entre disciplinas, la articulación entre saberes para un desarrollo sostenible y armónico de la sociedad (Lazo, 2011), se evidencia que la organización monodisciplinaria de los planes de estudio constituye un obstáculo para la formación interdisciplinaria (Perdomo, et al., 2014).

Por lo que se considera que si los profesores 
emplearan estrategias dentro del proceso formativo en el que se evidencien los vínculos interdisciplinarios del saber, se potenciaría el desarrollo científico en los estudiantes. Este último se interpreta como un proceso que promueve el pensamiento innovador, preparándolos para comprender los problemas de manera integral, cumpliendo el encargo de los contextos sociales en los que se han de desenvolver. Para ello, los docentes deberán lograr un equilibrio entre sus saberes disciplinares, pedagógicos y los tecnológicos $\mathrm{y}$, sobre todo, la capacidad y disposición de trabajar en un proceso social colectivo, dialógico y horizontal.

Para Royero (2007), la utilización de las redes de investigación constituye una vía que permite desarrollar los métodos científicos y tecnológicos de forma universal como base de la articulación interdisciplinaria, en el desarrollo de los métodos de investigación. Las equivocaciones que se producen con los enfoques fragmentados en el manejo del talento humano provocan dificultades en la toma de decisiones, por lo que se hace necesario que las diversas disciplinas colaboren e intercambien entre sí.

Los profesores que se identifican con el trabajo en equipo tendrán la capacidad de crear un escenario pedagógico sustentado en la interdisciplinariedad y en la integración de saberes, para poder cumplir con el encargo de la sociedad, ajustando sus metas y procedimientos a las demandas actuales.

Los actuales problemas necesitan ser profundizados a partir de diferentes disciplinas $\mathrm{y}$ formando equipos interdisciplinarios de trabajo, para que se puedan lograr soluciones sistémicas e integradoras (Escobar, 2010). Al respecto, los proyectos integradores buscan resolver desde el proceso de formación, problemas del contexto, a partir de la integración de personas, informaciones, conocimientos (teoría y práctica), áreas, metodologías activas de aprendizaje e investigación interdisciplinaria, para generar soluciones que contribuyan con el desarrollo social, a través de la relación universidadempresa (González, 2010).
De este modo, dichos proyectos pueden constituirse en una vía efectiva para que los estudiantes desarrollen actitudes para trabajar y efectuar el análisis de los problemas del contex to de forma integral y no individualizada, siendo las TIC (a partir de las múltiples posibilidades que ofrecen) un medio importante para lograrlo. Al respecto, para la Universidad de Oviedo las herramientas de la web 2.0 pueden favorecer la creación de proyectos integradores que vinculen varias disciplinas, "[...] propiciando un enriquecimiento mutuo a partir del flujo constante de información y de la participación en experiencias que redundan en la construcción compartida del conocimiento".

Puede decirse entonces que las TIC promueven sitios virtuales de comunicación, de intercambio de ficheros, de debate (Badia y García, 2006), fomentando el trabajo colaborativo para la solución de problemas de su entorno, lo que convierte al estudiante en un ser activo, propositivo y responsable. El empleo de las TIC en los procesos formativos obedece a la utilización que se haga de las mismas, por lo que es necesario emplearlas de manera efectiva, de tal modo que contribuyan a desarrollar la creatividad e independencia de los estudiantes, intensificando el rol orientador de los profesores. Esto exige que los primeros conozcan todas las posibilidades que éstas ofrecen y las usen eficientemente (Prieto, et al., 2011).

\section{Conclusiones}

Este ejercicio investigativo nos plantea que se requiere una educación superior que potencie la formación de las capacidades transformadoras humanas en los sujetos que participan en procesos formativos universitarios, con el empleo de tecnologías acordes al nivel del progreso social, que potencien niveles de desarrollo auténticos y que a la vez transformen sus contextos e incorporen la cultura universal. El análisis epistemológico referido ha revelado el insuficiente tratamiento didácticometodológico del vínculo indispensable de dichas tecnologías. La mirada epistemológica 
que se defiende, por una parte, direcciona su análisis hacia el perfeccionamiento de la dinámica de la formación investigativa de los estudiantes universitarios, con el empleo de las TIC, a partir de la concepción de proyectos integradores los que tienen a la interdisciplinariedad en su centro y están sustentados en el uso intensivo de dichas tecnologías.

La dinámica entonces constituye la parte "viva" de todo proceso formativo, ya que a través de la actividad y la comunicación entre los sujetos participantes en el mismo y el empleo de diversos métodos, se potencia en éstos el desarrollo de competencias que los preparan para la vida.

\section{Referencias}

Agazzi, E. (2004). El desafío de la interdisciplinariedad: dificultades y logros. Revista Empresa y Humanismo 2(2), 241252. Recuperado de http://dspace.unav.es/ dspace/bitstream/10171/5877/1/EVANDRO AGAZZI.pdf.

Aldana, G. (2012, febrero-mayo). La formación investigativa: su pertinencia en pregrado. Revista Virtual Universidad Católica del Norte, 35, 367-379. Recuperado de http://revistavirtual.ucn.edu.co/index.php/ RevistaUCN/article/view/366/701.

Álvarez, M. (2002). Acercamiento a la interdisciplinariedad en la enseñanza aprendizaje de las ciencias. La Habana: Editorial Pueblo y Educación.

Ander Egg, E. y Follari, R. (1988). Trabajo social e interdisciplinariedad. Recuperado de http://books.google.com/ books?id=Tc1JAAAAYAAJ\&pgis $=1$

Ávila, F. (2014). Aproximaciones a dinámicas de formación investigativa: experiencias en instituciones de educación superior en Colombia. Recuperado de http://www.oei.es/ congreso2014/memoriactei/1593.pdf.
Badia, A. y García, C. (2006). Incorporación de las TIC en la enseñanza y el aprendizaje basados en la elaboración colaborativa de proyectos. Recuperado de http://www.redalyc. org/pdf/780/78030211.pdf.

Bernal, C. (2014). Metodología de la investigación: para administración, economía, humanidades y ciencias sociales. México: Pearson Educación. Recuperado de https:// books.google.com.cu/books?id=h4X_eFai59 oC\&pg $=$ PA50\&lpg $=$ PA50\&dq $=$ integraci $\% \mathrm{C} 3$ $\% \mathrm{~B} 3 n+d e+$ saberes + que + generen + un + saber + superior $+a l+$ existente $+o+$ una + nueva + ciencia ${ }^{+} \mathrm{o}+$ disciplina + cient $\% \mathrm{C} 3 \%$ ADfica\&source $=\mathrm{b}$ 1\&ots=vUIFo5ujw5\&sig=ik7WF1_0qtmttmR J97IcSIPpMww\&hl=es

Blanco, O. et ál. (2011). El método científico y la interdisciplinariedad en el abordaje del Análisis de la Situación de Salud. Educación Médica Superior, 25(2), 29-39. Recuperado de http://scielo.sld.cu/scielo.php?script=sci arttext\&pid=S0864-21412011000200003\&ln $\mathrm{g}=\mathrm{es} \& \mathrm{nrm}=\mathrm{iso} \& \mathrm{t} \operatorname{lng}=\mathrm{es}$.

Botero, C. (2007). Los ejes transversales como instrumento pedagógico para la formación de valores. Recuperado de http:// www.gestiopolis.com/ejes-transversalesinstrumento-pedagogico-para-formacionvalores/.

Cabero, J. (2007). Las necesidades de las TIC en el ámbito educativo: oportunidades, riesgos y necesidades. Recuperado de http:// investigacion.ilce.edu.mx/tyce/45/articulo 1. pdf.

Cabezas, M. y Meriño, F. (2011). La interdisciplinaria en la formación inicial del maestro primario. Cuadernos de Educación y Desarrollo, 29(3). Recuperado de http://www. eumed.net/rev/ced/29/csmb.htm.

Chirino, M. V. et ál. (2005). El trabajo científico como componente de la formación inicial de los profesionales de la educación. La Habana: Editorial Cubana, MINED. 
Coll, C., Sarabia, B. y Valls, E (1992). Los contenidos en la reforma. Madrid: Editorial Alianza Psicología.

Delors, J., et al. (1996). La educación encierra un tesoro. Informe a la UNESCO de la Comisión Internacional sobre educación para el siglo XXI. Madrid: Ediciones UNESCO.

Díaz, F. y Hernández, G. (2004). Estrategias docentes para un aprendizaje significativo. Una interpretación constructivista. México: McGraw-Hill Interamericana.

Encarnación, E. (2010). El desarrollo de la interactividad cognitiva en entornos virtuales de enseñanza aprendizaje en el ámbito universitario. Tesis en opción al grado científico de Doctor en Ciencias Pedagógicas. Cuba: Universidad de Camagüey.

Escobar, Y. (2010). Interdisciplinariedad: desafío para la educación superior y la investigación. Revista Luna Azul. Recuperado de: http://200.21.104.25/lunazul/downloads/ Lunazul31_11.pdf

Ferry, G. (1997). Pedagogía de la formación. Buenos Aires: Ediciones novedades educativas.

Fiallo, J. (2012). ¿Cómo formar un pensamiento interdisciplinario desde la escuela? La Habana: Editorial Pueblo y Educación.

Fuentes, H., et al. (2011). La formación en la educación superior desde lo holístico, complejo y dialéctico de la construcción del conocimiento científico. CEES "Manuel F. Gran". Santiago de Cuba: Universidad de Oriente.

García, O (2010). Concepción pedagógica de un entorno virtual de enseñanza-aprendizaje desarrollador para la formación de profesores. Tesis en opción al grado científico de Doctor en Ciencias Pedagógicas. Santiago de Cuba:
Universidad de Ciencias Pedagógicas "Frank País García".

Godofredo, S. (2008). Interdisciplinariedad en la investigación social. Boletín informativo del programa de investigación estratégica en Bolivia. Recuperado de http://www.alertaspieb.com/UserFiles/File/PDFs/NEXOS-31. pdf.

González, H. (2011, julio-diciembre). Formación investigativa para la educación superior desde una perspectiva pedagógica. Revista Científica, 14, 72-78. Recuperado de http://cic.corhuila.edu.co/sitio/files/ Formaci\%C3\%B3nDeInvestigadores/ formacin_investigativa_para_la_educacin_ superior.pdf.

Hayes, H (2004). Workshop: Interdisciplinary Learning in Your Classroom. https://serc. carleton.edu

Hilarraza, Y. (2012). La investigación pedagógica: un aporte para la gestión de la formación docente desde un punto de vista socio cultural. Recuperado de http://dialnet. unirioja.es/servlet/articulo? codigo $=4228367 \&$ info=resumen\&idioma $=$ SPA.

Horruitiner,P. (2008). La universidad cubana: el modelodeformación.LaHabana:Editorial Félix Varela. Recuperado de http://books.google. com/books?id=TclJAAAAYAAJ\&pgis $=1$.

Izquierdo, J. M. (2004). La Gestión Académica del proceso docente educativo en la educación superior sustentada en las Tecnologías de la Información y las Comunicaciones. Tesis en opción al Grado Científico de Doctor en Ciencias Pedagógicas. CEES "Manuel F. Gran". Santiago de Cuba: Universidad de Oriente.

Jacob, W. J. (2015). Interdisciplinary trends in higher education. Palgrave Communications, 15001, 1-15. Recuperado de http://www.palgrave-journals.com/articles/ palcomms20151. 
Jiménez, W. (2006). La formación investigativa y los procesos de investigación científico-tecnológica en la Universidad Católica de Colombia. Recuperado de http:// repository.ucatolica.edu.co:8080/jspui/ bitstream/10983/474/1/Stud_1-1_A06_ Formaci $\% \mathrm{C} 3 \% \mathrm{~B} 3 \mathrm{n}$ investigativa.pdf.

Lazo, M. (2011). La interdisciplinariedad y la integralidad una necesidad de los profesionales de la educación. Recuperado de http://www. eumed.net/rev/ced/27/malp.htm.

López, L., Montenegro, M. y Tapia, R. (2005). La investigación, eje fundamental en la enseñanza del derecho. Guía práctica. Colombia: Publicaciones de la Universidad Cooperativa de Colombia. Recuperado de www.quadernsdigitals.net/index.php?accion. pdf.

Mauri, I (1993). Los contenidos escolares. Innovación Educativa. Madrid: Ediciones Morata.

Orrego, C. (1997). Cultura investigativa en acción. Publicación institución universitaria CEIPA. Recuperado Julio 9, 2007, de http:// www.ceipa.edu.co/ceipa/subsitios/lupa empresarial/ediciones_anterioran/edici on_05397.pdf

Pardo, M.E (2004). Las Tecnologías de la Información y las Comunicaciones en la dinámica del proceso docente educativo en la educación superior. Tesis presentada en opción al grado científico de Doctor en Ciencias Pedagógicas. Centro de estudios de educación superior "Manuel F. Gran" Santiago de Cuba: Universidad de Oriente.

Perdomo, et ál. (2014). Principios organizativos del conocimiento en un programa de enfermería. Recuperado de http:// www.redalyc.org/pdf/448/44831277009.pdf. interdisciplinaria de los profesores de ciencias: un ejemplo en el proceso de enseñanza aprendizaje de la física. Recuperado de http://karin.fq.uh.cu/ vladimar/ cursos $/ \% 23$ Did \%E 1 cticarrrr/Tes is Defendidas/Interdisciplinariedad/Leopoldo Fernando Perera Cumerma/Leopoldo Fernando Perera Cumerma.pdf.

Pérez, M., y Marreno, O. (2014). Competencias investigativas en la educación superior. Recuperado de http:// biblio.universidadecotec.edu.ec/revista/ edicionespecial/COMPETENCIAS $\% 20$ INVESTIGATIVAS\%20EN\%20LA.pdf.

Pizzul, M.E(2013). La formación humanística, fin esencial de la universidad. Argentina.

Polanco, C. (2015). Why interdisciplinary research matters. Nature, 525. Recuperado de http://www.nature.com/news/whyinterdisciplinary-research-matters-1.18370

Pozo, M. (2016). Dinámica formativa tecnoinvestigativa interdisciplinaria universitaria. Tesis presentada en opción al grado científico de Doctor en Ciencias Pedagógicas. Centro de estudios de educación superior "Manuel F. Gran". Santiago de Cuba: Universidad de Oriente.

Pozuelos, F., et ál. (2012). El enfoque interdisciplinar en la enseñanza universitaria y aprendizaje basado en la investigación. Un estudio de caso en el marco de la formación. Recuperado de http://www.redalyc.org/ pdf/2170/217028056012.pdf

Prieto, V. et ál. (2011). Impacto de las tecnologías de la información y las comunicaciones en la educación y nuevos paradigmas del enfoque educativo. Educación Médica Superior, 25(1), 95-102. Recuperado de http://scielo.sld.cu/scielo.php?script=sci arttext\&pid=S0864-21412011000100009\&ln $\mathrm{g}=\mathrm{es} \& \mathrm{nrm}=\mathrm{iso} \& \mathrm{t} \operatorname{lng}=\mathrm{es}$.

Perera, L. (2000). La formación Ramírez, R. (2013). Concepciones y modelos 
de la cultura investigativa en pregrado. IX Reunión Nacional de Currículo y III Congreso Internacional de Calidad e Innovación Universitaria. Recuperado de http://www. mecd.gob.es/dctm/revista-de-educacion/ numerosompletos/re355.pdf?documentId $=09$ $01 \mathrm{e} 72 \mathrm{~b} 811 \mathrm{c} 5825$.

Restrepo, B. (2001). Conceptos y aplicaciones de la investigación formativa y criterios para evaluar la investigación científica en un sentido estricto. Recuperado de http://www.cna.gov. co/1741/articles-186502_doc_academico5. pdf.

Rodríguez, N (2014). Dinámica formativa en telemedicina para las carreras de ciencias médicas. Tesis presentada en opción al grado científico de Doctor en Ciencias Pedagógicas. Centro de estudios de educación superior "Manuel F. Gran". Santiago de Cuba: Universidad de Oriente.

Royero, J. (2007). Las redes de I+D como estrategia de uso de las TIC en las universidades de América Latina. Recuperado de http:// www.uoc.edu/rusc/3/2/dt/esp/royero.pdf.

Ruiz, N et ál. (2011). La formación de los profesionales de la educación ante los retos de la educación superior contemporánea. Recuperado de http://www.cubaeduca.cu/ medias/pdf/2869.pdf

Salas, M. (2008). Modelo pedagógico de la dinámica del proceso de formación de gestores de programas y proyectos de ciencia e innovación. Tesis presentada en opción al grado científico de Doctor en Ciencias Pedagógicas. Centro de estudios de educación superior "Manuel F. Gran". Santiago de Cuba: Universidad de Oriente.

Sánchez, L (2009). Las Tecnologías de la Información y las Comunicaciones en la dinámica del proceso de formación para la investigación científica en la educación superior. Tesis presentada en opción al grado científico de Doctor en Ciencias Pedagógicas.
Centro de Estudios de Educación Superior "Manuel F. Gran". Santiago de Cuba: Universidad de Oriente.

Silva, J (2014). Dinámica Tecno-axiológica profesional. Tesis presentada en opción al Grado Científico de Doctor en Ciencias Pedagógicas. Centro de Estudios de Educación Superior "Manuel F. Gran". Santiago de Cuba: Universidad de Oriente.

Tapia, H (2015). Dinámica Tecno-formativaprofesional en entornos virtuales de aprendizaje. Tesis presentada en opción al Grado Científico de Doctor en Ciencias Pedagógicas. Centro de Estudios de Educación Superior "Manuel F. Gran". Santiago de Cuba: Universidad de Oriente.

Tejada, I. (2010). Evaluación de competencias profesionales en estudiantes de ingeniería de sistemas de información asistida por las tecnologías de la información y la comunicación. Tesis presentada en opción al grado científico de Doctor en Ciencias Pedagógicas. Cuba: Universidad de Camagüey.

Torres, A (2014). La construcción de las estrategias de aprendizaje en la formación inicial del profesional de la educación desde los entornos virtuales de aprendizaje. Tesis presentada en opción al grado científico de Doctor en Ciencias Pedagógicas. Universidad de Ciencias Pedagógicas "Frank País García". Santiago de Cuba: Universidad de Oriente.

Torres, J. (1994). Globalización e interdisciplinariedad: el curriculum integrado. Recuperado de http://books.google.com/ books?id=A3hUd70u0wAC\&pgis $=1$.

UNESCO. (1995). Documento de política para el cambioy desarrollo en la Educación Superior. París, Francia. Universidad de Oviedo. (2007). Jornadas de Intercambio de Experiencias en Docencia Universitaria en la Universidad de Oviedo. Recuperado de https://books.google. com.cu/books?isbn=84831762622007. 
Wojcipchowski, F. (2004). What is Interdisciplinary Research? National Academies. Washington: National Academy Press, p. 2. Recuperado de https://www. nsf.gov/od/oia/additional_resources/ interdisciplinary_research/definition.jsp 